\title{
Evaluation of orientation strategies in laparoscopic cholecystectomy
}

Mikael H Sodergren MRCS ${ }^{1}$, Felipe Orihuela-Espina $\mathrm{PhD}^{1,2}$, James Clark MRCS $^{1}$, Julian Teare FRCP ${ }^{1}$, Guang-Zhong Yang PhD ${ }^{2}$, Ara Darzi KBE ${ }^{1}$

${ }^{1}$ Department of Biosurgery and Surgical Technology, Imperial College London, St.Mary's Hospital, South Wharf Rd, Paddington, W2 1NY, UK

${ }^{2}$ Institute of Biomedical Engineering, Imperial College London, South Kensington Campus, London, SW7 2AZ, UK

Corresponding author (reprints will not be available from author):

Mr Mikael H Sodergren MBChB MRCS (Eng)

Department of Biosurgery and Surgical Technology

Imperial College London

Academic Surgical Unit

$10^{\text {th }}$ Floor QEQM

St.Mary's Hospital

South Wharf Road

London

W2 1NY

Telephone: $\quad+44(0) 2078866840$

Fax $\quad+44(0) 2078866309$

E-mail m.sodergren@imperial.ac.uk

Keywords: orientation, strategy, laparoscopic cholecystectomy, surgery, disorientation

There were no sources of funding or grants specifically received for this research. Mikael Sodergren holds a BRC clinical fellowship grant supporting a wider research project.

Running head: Orientation strategies in laparoscopic cholecystectomy 


\section{Introduction}

Laparoscopic surgery has revolutionized the way we perform many operative interventions, however with the benefits of a decrease in patient morbidity and reduced lengths of stay come a new set of operative problems experienced by the surgeon. These are almost exclusively related to the operating platform and the fact that operations are performed using a 2 dimensional monitor as interface for visual sensory input with the associated change in haptic feedback from the instruments used. The main differences this introduces compared to open surgery are related to changes in depth perception, a limited field of view, as well as an assistant on the whole operating the camera whilst the primary surgeon controls the operative instruments, amongst other things.

Laparoscopic cholecystectomy was the first widely adopted application of laparoscopic techniques in general surgery. It is now the gold-standard approach to this operation and remains one of the most commonly performed laparoscopic procedures world-wide. Up to 700,000 laparoscopic cholecystectomies are performed yearly in the USA ${ }^{1}$. One of the most important complications of this operation is major bile duct injury, which is associated with a mortality of $3 \%^{2}$, a complication rate of $26 \%{ }^{3}$ and significant associated morbidity and healthcare costs. The rate of major bile duct injury has remained in the range of $0.3-0.5 \%$, with most injuries going unrecognised at the time of surgery, although a recent study from the Netherlands has reported rates of up to $1 \%{ }^{4}$. It is known that the major cause of bile duct injury is misidentification of the biliary anatomy, and because 3 out of 4 injuries are not recognised intra-operatively it has been suggested that traditional surgical teaching provides inadequate reference points to prevent duct misidentification and that the most significant factor is spatial disorientation on the part of the surgeon ${ }^{5}$. Spatial disorientation could, for instance, lead to a surgeon dissecting on the medial side of the common bile duct, mistakenly believing that the cystic duct is being displayed ${ }^{6}$. Way et $\mathrm{al}^{7}$ demonstrated that the errors leading to laparoscopic bile duct injury arise on the whole from a visual perceptual illusion rather than errors in skill, knowledge or judgement. It is impossible to calculate the proportion of bile duct injuries resulting from operator spatial disorientation since this is difficult to quantify retrospectively. The operator may not even have been aware he was disorientated, 
or may have been mistaken in the reorientation process however it is likely to account for substantial proportion of this type of surgical complication. Spatial disorientation is furthermore difficult to rectify when the operator is unaware they are disorientated, and this will be elaborated further in the discussion. However, when the operator does become aware of this, their ability to reorientate correctly and effectively may play a major role in the outcome of the operation. Little is currently known about the factors that influence a surgeons reorientational abilities including whether this is related to laparoscopic experience and therefore seniority. No studies to date have examined or described specific strategies used for reorientation during this operation or discussed the relation to outcome. This research is expected to benefit both trainee surgeons as well as experienced surgeons that consistently perform below average.

To obtain specific sensory information about our environment we have to focus our senses on that particular feature, therefore, the notion of focus of attention has a derived meaning referring to the physically observable behaviour of orientation towards an object. This may take the form of posture, head orientation and/or gaze. Gaze direction can therefore be used as an indicator of attention in a visual environment ${ }^{8}$. It is now widely accepted that distribution of eye fixations are governed by intention and can therefore be related to attention ${ }^{9}$ provided that the eye movement is task-relevant as defined by Kahneman ${ }^{10}$. Eye tracking data therefore allows a reliable identification of salient image features ${ }^{11}$ that can be mapped back to spatial space for highlighting regions of interest and attention selection ${ }^{12}$, and represents a method of knowledge gathering for decision support in image understanding ${ }^{13}$. By studying a surgeons gaze in a situation where he or she is attempting to orientate may therefore provide clues of visual attention and strategies used during this process.

The aims of this study were to examine if there are common patterns of visual attention strategies employed by surgeons which are associated with a greater chance of successful reorientation when disorientated during laparoscopic cholecystectomy, and to determine if seniority or laparoscopic experience influence success at reorientation. 


\section{Methods}

\section{Subjects}

The study was approved by the St.Mary's Research Ethics Committee (Ref: 08/H0712/104). All subjects were provided with an information leaflet and signed an appropriate consent form prior to commencement of the study. 21 surgeon subjects were recruited to the study (19M: 2F). Mean age was 31.6 yrs (range $28-38 \mathrm{yrs}$ ). There were 4 attending, 3 senior residents and 14 junior residents. Table 1 illustrates the demographics and operative experience of the surgeons.

\begin{tabular}{lccc} 
& $\begin{array}{c}\text { Mean number of } \\
\text { Mean } \\
\text { age }\end{array}$ & $\begin{array}{c}\text { Mean number of } \\
\text { laparoscopic } \\
\text { operations as } \\
\text { primary surgeon }\end{array}$ & $\begin{array}{c}\text { assisted } \\
\text { laparoscopic } \\
\text { operations }\end{array}$ \\
\hline $\begin{array}{l}\text { Junior } \\
\text { Residents }\end{array}$ & 30 & 15 & 94 \\
$\begin{array}{l}\text { Senior } \\
\text { Residents }\end{array}$ & 34 & 107 & 250 \\
Attendings & 36 & 475 & 1400
\end{tabular}

Table 1 Demographics and laparoscopic experience of subjects

\section{Experimental design}

During the experiment subjects were shown a series of 8 sequential images taken during human laparoscopic cholecystectomy operations from the operating laparoscope. The images represent typical views encountered during initial stages of a laparoscopic cholecystectomy up until division of the cystic duct divided into four image groups:

1. Overview of right upper quadrant structures

2. Close-up of right upper quadrant structures

3. Close-up of right upper quadrant structures with gallbladder retracted

4. Dissection of Calot's triangle 
The images used for this experiment are illustrated in fig 1. Three image groups contain the same images in various rotational transformations. The regions of interest of each image and their rotational orientation are given in table 2.

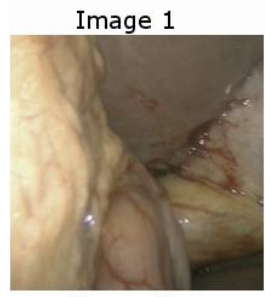

Image 5

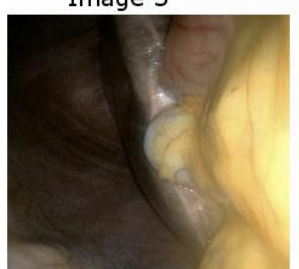

Image 2

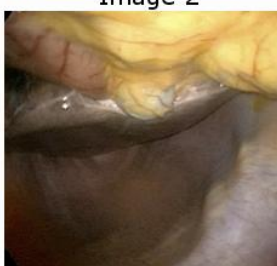

Image 6

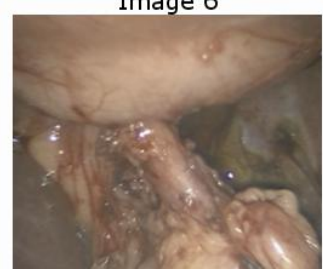

Image 3

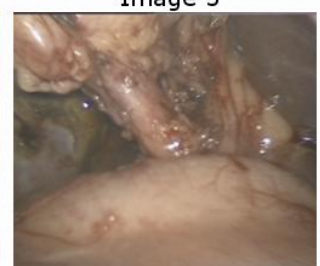

Image 7

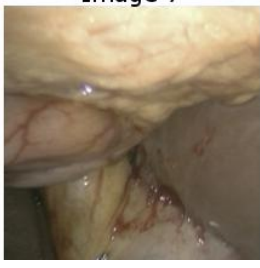

Fig 1 Visual stimuli used for experiment

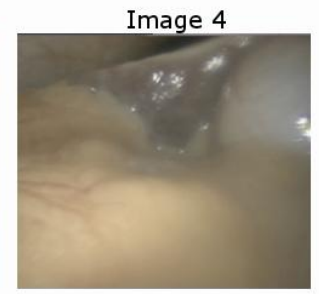

Image 8

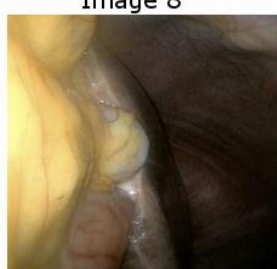

\begin{tabular}{|c|c|c|c|c|}
\hline $\begin{array}{l}\text { Image } \\
\text { Group }\end{array}$ & Stage of operation & Images & Regions of Interest & Orientation of Image \\
\hline 1 & $\begin{array}{l}\text { Overview of RUQ } \\
\text { structures }\end{array}$ & $\begin{array}{l}2 \\
5 \\
8\end{array}$ & $\begin{array}{l}\text { GB, Peritoneum, Stomach, } \\
\text { Omentum, Liver }\end{array}$ & $\begin{array}{l}2-180^{\circ} \\
5-90^{\circ} \text { anti-clockwise } \\
8-90^{\circ} \text { clockwise }\end{array}$ \\
\hline 2 & $\begin{array}{l}\text { Close-up of RUQ } \\
\text { structures }\end{array}$ & 4 & $\begin{array}{l}\text { GB, Peritoneum, Liver, } \\
\text { Omentum }\end{array}$ & 4 - Normal \\
\hline 3 & $\begin{array}{l}\text { Close-up of RUQ } \\
\text { structures with GB } \\
\text { retracted }\end{array}$ & $\begin{array}{l}1 \\
7 \\
7\end{array}$ & $\begin{array}{l}\text { GB, Liver, Stomach, } \\
\text { Omentum, Calot's triangle } \\
\text { (undissected) }\end{array}$ & $\begin{array}{l}1-90^{\circ} \text { clockwise } \\
7-189^{\circ}\end{array}$ \\
\hline 4 & $\begin{array}{c}\text { Dissection of Calot's } \\
\text { triangle }\end{array}$ & 6 & $\begin{array}{l}\text { GB fundus, Cystic duct, } \\
\text { Calot's triangle } \\
\text { (dissected), Lesser } \\
\text { Omentum, Stomach }\end{array}$ & $6-180^{\circ}$ \\
\hline
\end{tabular}

GB- Gallbladder RUQ- Right upper quadrant

Table 2 - Image groups, stage of operation, relevant regions of interest in each group and orientational transformation as seen in fig 1

The aim was to disorientate the subjects with the introduction of the image and to observe reorientation behaviour. The images could only have been manipulated on a rotational axis in 
increments of $90^{\circ}$ from a "normal" view (where contour of liver parallel to the horizontal or cystic duct almost vertical in dissection of Calot's triangle). The "normal" view is the image orientation preferred by the vast majority of laparoscopic surgeons during this operation and this was described and illustrated with an unrelated laparoscopic image in the subject information sheet prior to commencement of the study. The task was to determine how, or if the images were manipulated with respect to their orientation. There was no time limit imposed on subjects to provide an answer for each image. Subjects controlled the progression of images via a keyboard and were given time between images to record answers on a sheet provided. Subjects were not told if their answers were correct or incorrect.

\section{Eye-tracking}

Gaze tracking was performed using a Tobii ET 1750 eye tracker (Tobii Inc, Sweden). This is an infrared video-based binocular eye-tracking system recording the position of gaze in the work plane (screen) at $30 \mathrm{~Hz}$. Infra-red light source is beamed towards the eye whilst a camera records the position of the reflection (known as the Purkinje reflection) on the cornea surface relative to the pupil centre. The infra-red images are real-time digitized and processed. Following a calibration procedure ${ }^{14}$, the point of regard can then be determined with an accuracy of 1 degree across the work plane. This allows accurate tracking of the position of gaze of subjects standing approximately $60 \mathrm{~cm}$ away from the equipment looking at the monitor. Fixations were detected using a dwell time algorithm by which a fixation is considered when the gaze does not move more than a hard threshold (30 pixels), during a minimum of a $100 \mathrm{~ms}^{14}$.

As the participants are shown each new image stimulus to discern its orientation, their eyegaze navigates between the organs and structures in the scene exposing differences in visual behaviour. We analysed these differences in visual behaviour using an algorithm which has been detailed and validated to use for the purpose of comparing visual reorientation behaviour previously ${ }^{15,16}$. The output of this algorithm produces a 2 dimensional graph plotting the behaviour of each subject as a single point. Points in close proximity in the output plot are shown to demonstrate similarity in visual reorientation behaviour and by examining 
gaze plots the significance of moving across the two axes can be inferred. The classic kmeans algorithm ${ }^{17}$ was used to compute the centroids for the subject groups who answered correctly in each image and the closest subject the centroids of this group was selected as the most representative to discern the average successful reorientation strategy for each image.

Behavioural Data

Each image or stimulus was manually segmented into regions of interest (ROIs) delimiting the different significant organs and structures. The outcome parameters of this study were time taken to provide each answer, whether the answer was correct / incorrect, and basic eyetracking parameters such as fixations and dwell-time per ROI. Other outcome parameters were fixation sequences on ROls as determined by organs and structures occupied in the scene and the order in which these were fixated upon. To normalize the dwell time per region of interest this was divided by the relative area occupied by that structure within each image.

Comparisons between groups of non parametric variables were done using the Kruskal Wallis test with individual variables compared using the Mann Whitney $U$ test as necessary. Correlations of continuous variables were determined by non-parametric linear regression. All analyses were performed using statistical software (SPSS v16.0, Chicago, USA). Differences were considered statistically significant at $p<0.05$.

\section{Results}

Overall orientation score

Junior residents, senior residents and attending surgeons answered an average of 6.36 (range 2-8), 6 (range 5-7) and 6.25 (range 5-7) out of 8 correctly respectively. There was no statistically significant difference in number of correct answers between these groups $(p=.919)$. There was no significant correlation between number of previously performed 
laparoscopic operations as first surgeon $\left(p=.78 ; r^{2}=-0.065\right)$ or assistant $\left(p=.257 ; r^{2}=-0.259\right)$ with the number of correct answers given (see fig 2). 8 subjects were aware that they adopted a specific reorientation strategy. There was no difference in number of correct answers given between subjects who declared they were aware of a reorientation strategy and those who did not $(p=.795)$.
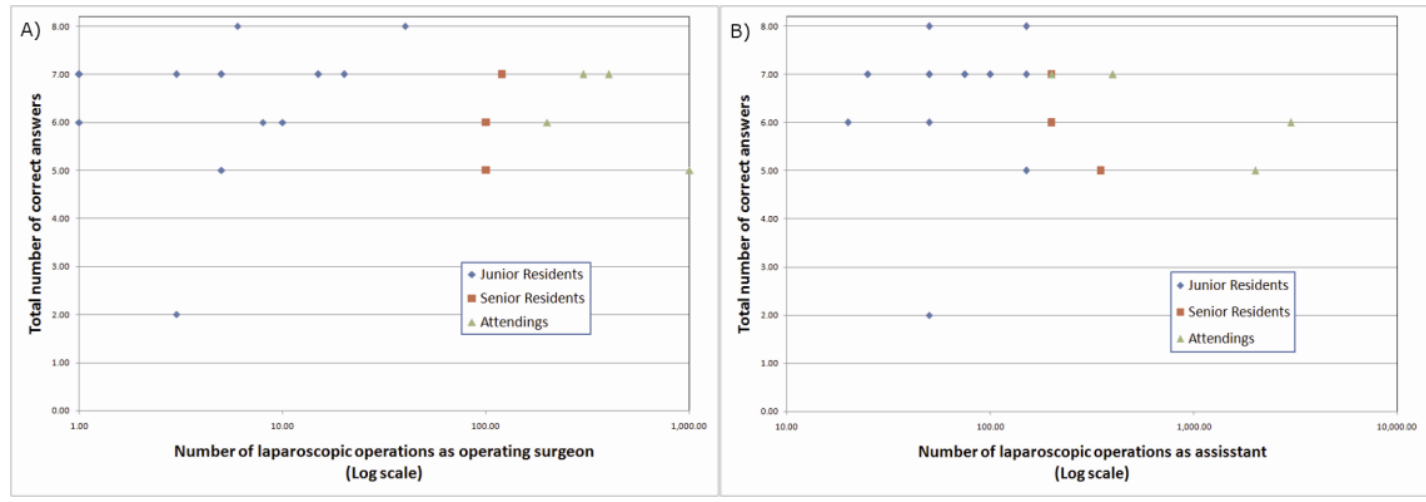

Fig 2 - scatter plot of number of correct answers against number of laparoscopic operations as a) operating surgeon and b) assistant

Eye tracking parameters

Eye tracking parameters was analyzed from two perspectives as illustrated in tables 3 and 4:

1) seniority of subjects (3 groups)

2) number of correct answers given (5 groups)

\begin{tabular}{lcccc} 
& $\begin{array}{c}\text { Junior } \\
\text { Residents }\end{array}$ & $\begin{array}{c}\text { Senior } \\
\text { Residents }\end{array}$ & Attendings & P \\
\hline Mean time taken to answer (sec) & 20.9 & 18.2 & 12.4 & 0.174 \\
Mean number of fixations & 41.9 & 45.8 & 29.2 & 0.123 \\
Mean absolute dwell time / ROI (ms) & 2309 & 3197 & 1836 & 0.78 \\
Mean normalised dwell time (ms/pixels ${ }^{2}$ )/ROI & 1.99 & 2.3 & 2.1 & 0.997
\end{tabular}

Table 3 Comparison of basic eye tracking parameters by seniority of subject 
Number of Correct Answers (/8)

\begin{tabular}{lcccccc} 
& 2 & 5 & 6 & 7 & 8 & P \\
\hline \hline Mean time taken to answer (sec) & 15.5 & 19 & 16.5 & 20.4 & 21 & 0.174 \\
Mean number of fixations & 22.5 & 38.3 & 41 & 38.2 & 56.3 & 0.123 \\
Mean absolute dwell time / ROI (ms) & 1923 & 1774 & 2624 & 2273 & 2893 & 0.004 \\
Mean normalised dwell time $\left(\mathrm{ms} /\right.$ pixels $^{2}$ )/ ROI & 1.72 & 2.12 & 2.09 & 2.12 & 1.74 & 0.911
\end{tabular}

Table 4 Comparison of basic eye tracking parameters by total number of correct answers of subjects

It can be seen that when mean dwell time per ROI was normalized there was no difference in seniority or performance groups.

Since the performance between seniority groups were not significantly different for this study the mean normalised dwell time between all groups was used for further analysis. The dwell times on individual organs and structures has been analysed for different stages of the operation as detailed in table 1 .

Visual behaviour profiling \& average successful reorientation behaviour

It is naturally anticipated that individual surgeons may use different successful reorientation strategies but to discern a generalizable average successful behaviour the subject closest to center of gravity of the group who answered correctly was chosen as the most representative behaviour. The center of gravity represents the mean or central point of the cluster as computed by the k-means algorithm.

The image results will be presented below in the order in which they are encountered during the operation:

\section{1) Overview of right upper quadrant structures - Images 2, 5 \& 8}

As shown in fig $3 a$ and $3 b$ there appeared to be similar amounts of attention by dwell-time across the images indicating the use of a comparable and consistent orientation strategy across subjects and seniorities. During overview of right upper quadrant structures, surgeons focused heavily on the position of the gallbladder, liver and the stomach for reorientation. It 
appears that when the image is manipulated by $90^{\circ}$ the attention paid to the gallbladder increases to a maximum when the image is rotated clock-wise.

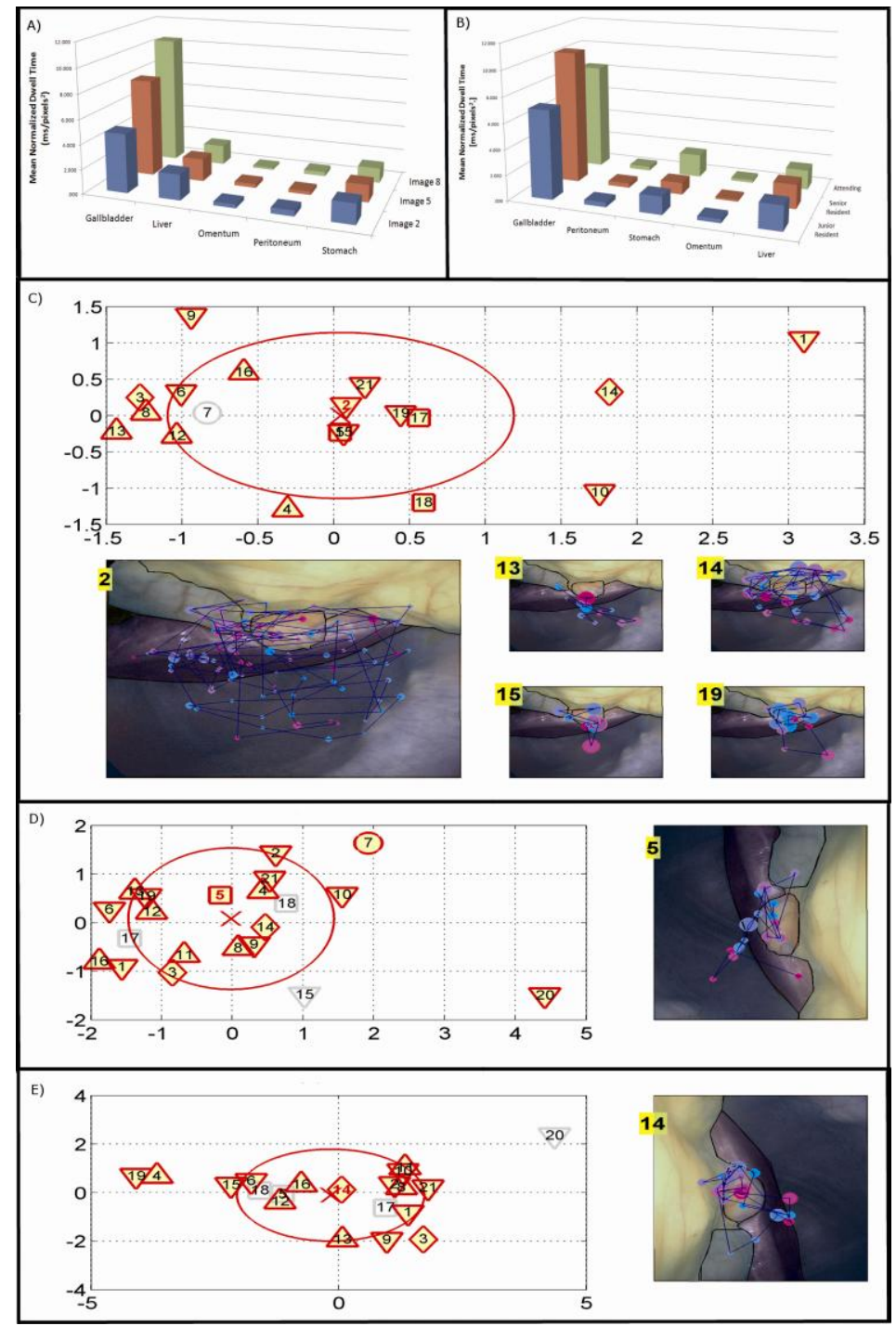

Fig 3a) Mean normalized dwell times per region of interest in stimulus images 2,5 \& 8. 3b) Mean normalized dwell times per region of interest for the image group per subject seniority. 3c) Example of the visual path profiling algorithm output illustrating subject performance (circle $=2$ total correct, squares $=5$, upward pointing triangle=6, downward pointing triangle=7 \& diamonds=8), and answer, (yellow shading=correct answer, no shading=incorrect answer) for the stimulus image 2. Subject numbers are represented within shapes. Centroids are illustrated by cross in the middle of sphere for subjects who answered correctly. Closest subject identified by highlighting number red. Relevant gaze plots are shown for selected subjects with initial fixations coded in blue turning to red to the end of the fixation sequence. 3d) Output of visual path algorithm for stimulus image 5. Coding is as for fig 3c. 3e) Output of visual path algorithm for stimulus image 8 . Coding is as for fig 3c.

The profiling output in fig $3 c$ confirms the relative importance of the gallbladder as a visual cue for reorientation in this image as established by the high mean normalized dwell time 
displayed in fig 3a. The fact that most surgeons correctly orientated in this scenario is likely to reflect the familiarity of this view. The majority of subjects use the gallbladder as an "anchor object" and relate the relative positions of the liver and stomach to this during orientation. The majority of subjects additionally used the peritoneum of the abdominal wall and diaphragm and the omentum to a lesser extent as additional cues as seen by the gaze plots close to the center of gravity in fig 3c. By examining the gaze plots as we move across the abscissa axis in fig $3 c$ it can be seen that the gallbladder remains a central visual cue. As illustrated by gaze plots of subjects $13,15,2$ and then 14 by moving from left to right across this axis the focus of attention to peripheral reference structures changes from the peritoneum of the diaphragm and anterior abdominal wall to the greater omentum. It is important to highlight that the profiling plots capture both the spatial distribution of fixations as well as the temporal aspect of the order on which structures are fixated upon. To that end, it is possible to quantify the orientation strategy. In fig 3c the most representative behaviour is that illustrated by subject 2 however this fixation sequence is quite long and therefore difficult to quantify visually however subject 15 is very close to the center of gravity point and the strategy employed by this subject is easily discernable. Initially the gallbladder is identified and its position is related to the liver, stomach, the greater omentum and finally the peritoneum of the diaphragm. An almost identical strategy is displayed by subject 19 .

Fig $3 d$ clearly illustrates the strategy adopted by subject 5 in reorientation. This involves initial identification of the gallbladder and thereafter relating positions of liver, stomach and ultimately peritoneum of the abdominal wall to the position of the gallbladder to deduce correct orientation of the image. An almost identical strategy is displayed by subject 14 in fig 3e confirming the reliability of this methodology to evaluate successful behaviour.

\section{2) Close up of right upper quadrant structures -_Image 4}

As shown in fig $4 \mathrm{a}$ and $4 \mathrm{~b}$, it appears that when surgeons are confronted with a limited view of organs in the right upper quadrant, identification and orientation by the position of the liver is common and important with similarities of focus across seniority levels although attending surgeons appear to focus somewhat more on the gallbladder. Fig 4c illustrates the 
homogeneity of orientational behaviour which appears to focus heavily on the position of the liver relative to the gallbladder with some attention placed on relative positions of omentum. Comparatively little attention is paid to the peritoneum of the abdominal wall, probably reflecting the difficulty of using this structure as an effective visual cue in this scenario. The importance of attention to the liver and gallbladder corresponds well with the overall high mean normalized dwell times shown in fig $4 a$.

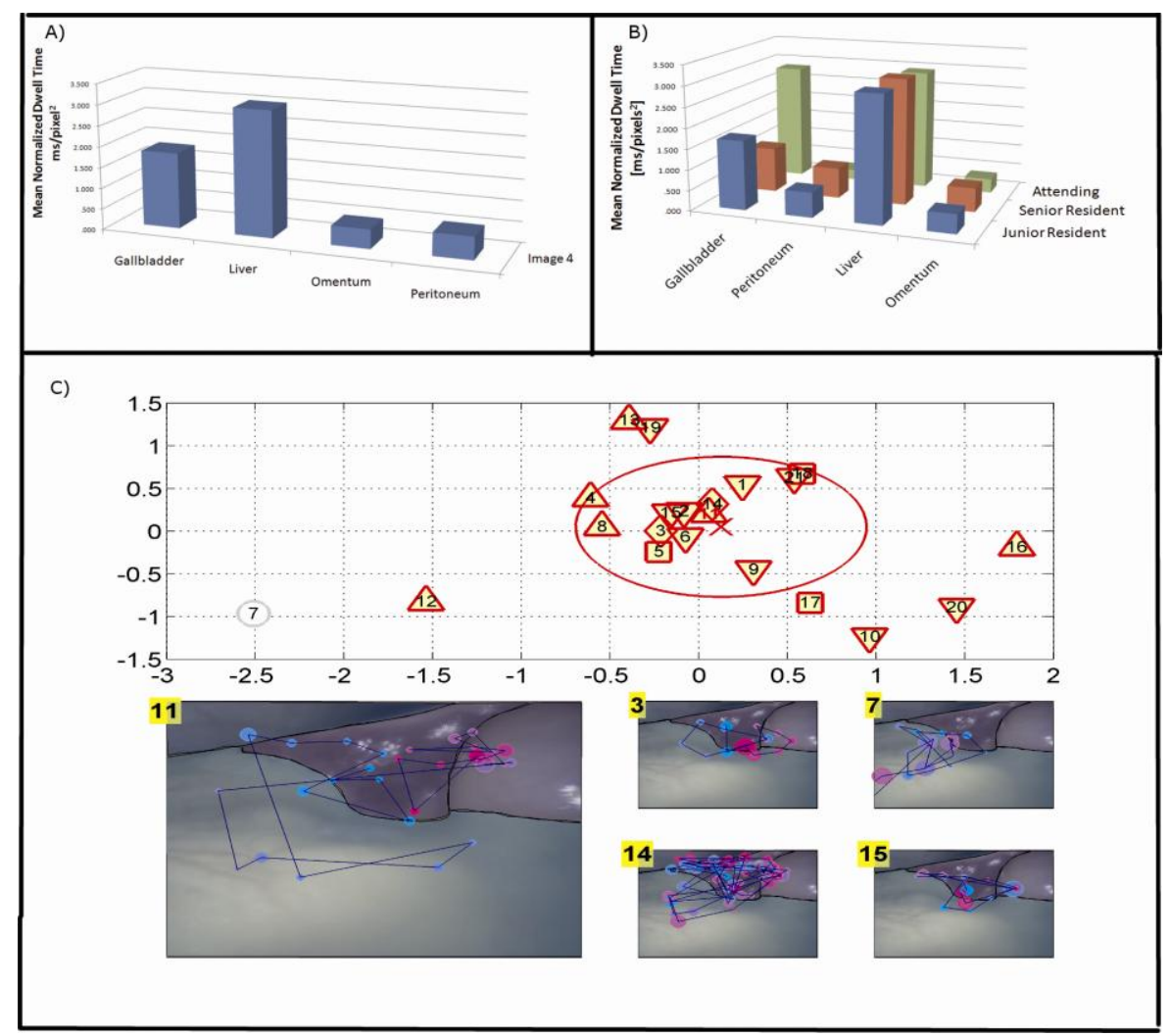

Fig 4a) Mean normalized dwell times per region of interest in stimulus image 4. 4b) Mean normalized dwell times per region of interest per subject seniority. 4c) Output of visual path algorithm for stimulus image 4. Coding is as for fig 3c.

Subject 11 clearly illustrates the average successful orientation strategy used starting by identifying the liver and relating relative positions of the gallbladder and other structures to this. Subject 7 adopted an unsuccessful reorientation strategy and the reason for this is revealed when examining the gaze plot. The liver and omentum were the only structures focused on by this subject with no attention paid to the position of the gallbladder. 


\section{3) Close-up of right upper quadrant structures with gallbladder retracted - Images 1 \& 7}

As shown in fig $5 \mathrm{a}$ the normalized dwell time profile is consistent across both images with similar dwell-time for Calot's triangle, the gallbladder and the stomach across both images with less attention paid to the liver and omentum. This again suggests that subjects used similar and coherent strategies for this view despite different image transformations. Fig $5 b$ shows similarities in dwell time profiles across seniorities.

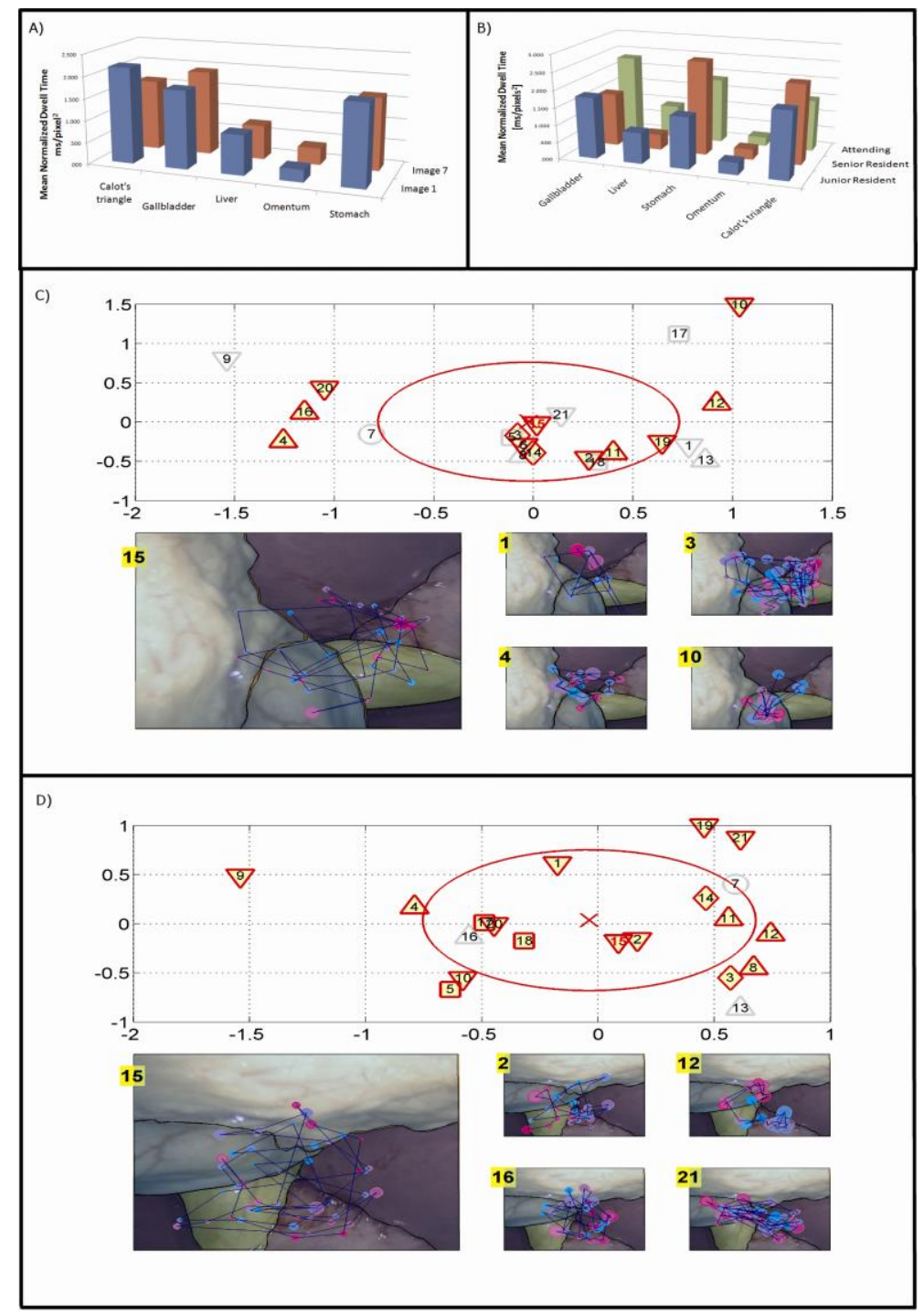

Fig 5a) Mean normalized dwell times per region of interest in stimulus images 1 and 7. 5b) Mean normalized dwell times per region of interest per subject seniority. 5c) Output of visual path algorithm for stimulus image 1. Coding is as for fig 3c. $5 \mathrm{~d}$ ) Output of visual path algorithm for stimulus image 7 . Coding is as for fig 3c. 
Fig $5 \mathrm{c}$ illustrates the visual behaviour profiling revealing the various reorientation strategies used in this scenario. Moving from left to right across the abscissa axis subject 4 prioritises the liver and omentum whilst on the other end of the axis subject 1 almost completely disregards the omentum as a reorientation cue but focuses heavily on liver, gallbladder and Calot's triangle. Subject 10 displays a unique strategy focusing heavily on the stomach as a visual re-orientational cue. Subject 3 lies close to the center of gravity of successful subjects and when examining the gaze plot it can be seen that this corresponds well with the overall mean normalized dwell times.

When examining fig $5 d$ the same trend becomes apparent. Moving across the abscissa axis shifts the subjects focus from the liver towards the gallbladder with decreasing amounts of fixations on the omentum. Subject 2 lies close to the center of gravity of successful subjects and the gaze plot also appears to correspond well with average dwell time behaviour with much focus on Calot's triangle, gallbladder and stomach in seemingly equal proportions with less attention on the omentum and liver. Subject 21 illustrates an uncommon behaviour whose reorientation strategy appears to completely ignore Calot's triangle.

The average successful reorientation behaviour is illustrated in fig $5 \mathrm{c}$ and $5 \mathrm{~d}$ which both show that the visual reorientation strategy of subject 15 was the closest to the mean behaviour of successful subjects in both images 1 and 7 . By looking at the gaze plots of this subject in both images it can be seen that an almost identical reorientation strategy was used in both images. This involved initial identification of liver, stomach, gallbladder, omentum and then Calot's triangle in turn followed by alternating fixations on mainly the gallbladder, Calot's triangle and stomach to deduce orientation.

\section{4) Dissection of Calot's triangle - Images 3 \& 6}

This group represents close-up images of a dissection of Calot's triangle with a clearly visible cystic duct. Subjects appeared to focus heavily on the cystic duct and relating this to the positions of the dissected Calot's triangle or the lesser omentum. The similarities in dwell time across both images and seniorities are again very apparent in figs $6 \mathrm{a}$ and $6 \mathrm{~b}$. 


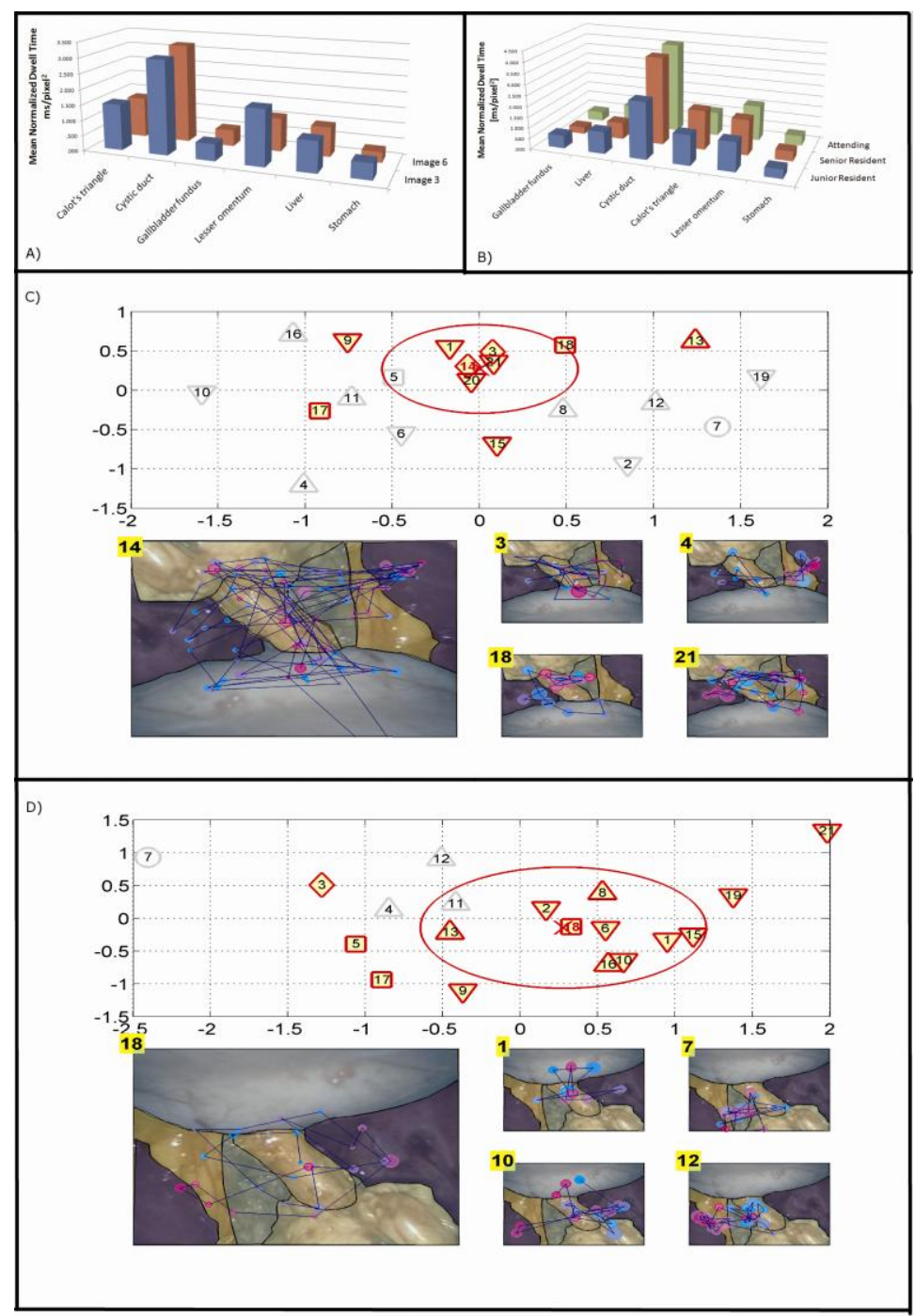

Fig 6a) Mean normalized dwell times per region of interest in stimulus images 3 and 6. 6b) Mean normalized dwell times per region of interest per subject seniority. 6c) Output of visual path algorithm for stimulus image 3. Coding is as for fig 3c. 6d) Output of visual path algorithm for stimulus image 6 . Coding is as for fig 3c.

In fig $6 \mathrm{c}$ there appears to be substantial clustering of subjects who answered the question correctly. On closer inspection of these gaze plots it is apparent that these subjects relied heavily on the cystic duct as a reorientation cue apparently using this as a reference to the position of Calot's triangle and the lesser omentum between the liver as clearly illustrated by the gaze plots of subjects $3,14,18 \& 21$. Subject 4 applied an unsuccessful strategy and it can be seen that there is much less focus on the position of the cystic duct and no attention given to Calot's triangle. 
A similar pattern of behaviour is observed in fig $6 \mathrm{~d}$. The visual behaviour of subject 12 illustrates an unsuccessful reorientation strategy with heavy focus on the position of the liver to the cystic duct, which of course would not allow differentiation between $180^{\circ}$ rotations.

Due to the relative complexity of this scenario the fixation sequences to orientate tended to be longer than in other scenarios, however using the gaze plots in fig $6 \mathrm{c}$ and $6 \mathrm{~d}$ such as that of subject 18 in combination with the information of the normalized dwell times per ROI, it is nevertheless possible to accurately describe a representative successful behaviour. This involved initial identification of the cystic duct, followed by identification and relation of the lesser omentum to the cystic duct, followed by the liver, stomach, Calot's triangle and finally gallbladder fundus to deduce orientation. It is apparent that after identification of a structure this is related by a subsequent fixation on the cystic duct used as "anchor" object.

From table 5 it can be seen that apart from the first image, when subjects were familiarising themselves with the experimental task and set-up, image group 4 (images $3 \& 6$ ) appears to be the most complex illustrated by higher number of fixations and event durations than other image groups.

Number of Correct Answers (/8)

\begin{tabular}{lcccccc} 
& 2 & 5 & 6 & 7 & 8 & $\mathbf{P}$ \\
\hline \hline Mean time taken to answer (sec) & 15.5 & 19 & 16.5 & 20.4 & 21 & 0.174 \\
Mean number of fixations & 22.5 & 38.3 & 41 & 38.2 & 56.3 & 0.123 \\
Mean absolute dwell time / ROI (ms) & 1923 & 1774 & 2624 & 2273 & 2893 & 0.004 \\
Mean normalised dwell time(ms/pixels ${ }^{2}$ )/ ROI & 1.72 & 2.12 & 2.09 & 2.12 & 1.74 & 0.911 \\
$\quad$ Table 4 Comparison of basic eye tracking parameters by total number of correct answers of subjects & &
\end{tabular}

\section{Discussion}

The results of this study indicate that there are discernable and quantifiable visual attention strategies used by surgeons during laparoscopic cholecystectomy associated with successful orientation. There was no difference in success in reorientation between different seniorities and laparoscopic experience of the surgeon subjects. This finding is corroborated by the lack of grouping of subjects in the visual profiling outputs between seniorities or laparoscopic 
experience. In some instances however, there was grouping of subjects who orientated successfully on a particular image, indicating similar individual strategies, as illustrated in fig 6c. Although performance results were homogeneous across seniority groups in this study, increased subject numbers or a greater breadth of experience between the subject groups may have demonstrated a difference in performance. Given the present results however, this was deemed unnecessary as the main aim of this study was to characterise effective behaviour. It is important to note that all subjects had significant laparoscopic experience as assistants if not as primary surgeons. The homogeneity of performance across experience levels suggest that after a certain level of laparoscopic experience surgeons reach a plateau consistent with their innate orientational abilities. This is consistent with the finding that bile duct injuries have appeared to remain constant over recent years despite improvements in surgical instruments and visualization equipment ${ }^{2}$. As it is thought that the majority of bile duct injuries occur as a result of spatial disorientation ${ }^{5}$, the implication of this is that the risk of bile duct injury as a result of disorientation is intrinsic to the operation due to the innate abilities of surgeons. It is therefore important to note that in this study even the most experienced surgeons were mistaken on average $22 \%$ of the time. This is not a trivial percentage and reflects the importance of this issue in relation to laparoscopic surgery.

As expected, there were individual differences in both visual attention strategies used and performance outcome. This correlates well with findings in the psychology literature which suggest there is a wide range of performance in relation to orientation with age and sex being contributing factors as well as the features of the spatial environment ${ }^{18-21}$.

It is intuitive that the initial fixations when presented with a novel scenario is to correctly identify the relevant anatomical structures, and the time taken and relative focus of this will differ between individuals. In this study we assume surgeons correctly identified most relevant anatomy and recognize that detection of a familiar scenery by an individual may only require a few carefully placed fixations. Nevertheless, this experiment attempts to discern behaviour after anatomy recognition when attention strategies are employed to achieve orientation. With this in mind, we showed that for all scenarios presented of a laparoscopic cholecystectomy there appeared to be a common strategy used by a large proportion of surgeons which was associated with a high chance of successful reorientation. The combination of images used in 
this study represents many of the common views faced by a surgeon during a laparoscopic cholecystectomy.

It was very apparent that choosing an "anchor object" and relating position of selected structures to this was an almost a uniformly successful orientation strategy. The choice of anchor object and related structures was different during various stages of laparoscopic cholecystectomy and this choice can determine the outcome of the orientation process.

It was clear that when viewing the organs of the right upper quadrant the relative position of the gallbladder as an "anchor object" to the liver and stomach was a very powerful and successful reorientational strategy. When the gallbladder was retracted the choice of anchor object became less clear and it appeared that either use of the gallbladder, stomach or the region of Calot's triangle as long as they were related to the position of the other two structures proved to be effective in reorientating. Surgeons who correctly reorientated when presented with an image of a dissected Calot's triangle almost exclusively used the position of the cystic duct as an "anchor object". This is unlikely to represent a featural cue as the position of the duct within the image will not correctly identify orientation however what appears important is likely to first be the geometry of the duct i.e. if the duct is relatively vertical the image must be normal in orientation or rotated $180^{\circ}$, and thereafter the relative position of the duct to surrounding structures such as the lesser omentum and stomach, which will differentiate between these transformations. The fact that graphs of mean normalized dwell times per $\mathrm{ROI}$ of the same image in different rotations are almost identical provides strong evidence that whatever the rotation of an image presented to a surgeon, the relative importance of ROI's are consistent and similar between transformations.

It was interesting to note that on questioning only a minority of surgeons (8/21) were actually aware that they adopted a particular reorientation strategy. This further supports the idea that this represents an automated phenomenon in the majority, which has been cognitively programmed through experience. This study assumed that surgeons did not have an advantage over each other with regards to their cognitive map, which in this case represents their knowledge of the correct anatomy concerned with the operation, as all subjects had 
completed specific surgical postgraduate examinations containing relevant surgical anatomy and have operative experience in relation to laparoscopic cholecystectomy.

Analysing a combination of overall normalized dwell times per $\mathrm{ROI}$ and the average successful fixation sequences have allowed us to accurately describe successful orientation strategies.

In preliminary studies, reorientation was investigated in a more diverse population of subjects, without a previously formed cognitive map of the scenery prior to the experiment, and not necessitating the use of knowledge of human anatomy ${ }^{15}$. This showed a more varied difference in performance between individuals but highlighted some common strategies. When this experiment was applied to laparoscopic surgery as in this study, successful reorientation appeared to be more prevalent reflecting the familiarity of this paradigm and task to many surgeons.

It is important to point out that using eye-tracking for research into human attention processes carries some known assumptions and limitations. Because attention is composed of both higher and lower level functions it is well known that humans can voluntarily dissociate attention from their foveal direction of gaze $\mathrm{e}^{22}$. This high level component of vision is obviously much more difficult to detect by objective external observation. Therefore, the important assumption that attention is linked to foveal point of gaze is usually made in eye-tracking experiments. We believe that this assumption holds true especially if the right experimental conditions are met. In this experiment we believe this is the case because visual attention selection is driven by a top-down mechanism and is therefore derived from task specification i.e. the orientation process, rather a reactive mechanism caused by salient stimuli (bottomup). The eye tracking equipment used in this study is a remote video-oculography (VOG) based system integrated with a normal computer screen, and therefore does not change the normal navigation behaviour of the subjects. It can cater for head movement and there were no concerns from subjects regarding the adequacy of the equipment. It is worth noting that like all existing state-of-the-art eye-tracking systems, our setup does require a subject specific calibration step at the beginning of the experiment. Our research group are developing techniques that can significantly simplify or potentially remove this step. 
Disorientation and therefore patient morbidity may occur in a number of scenarios during this operation. Firstly, the operator may not be aware they are disorientated. This may happen if the anatomy encountered is not normal. Secondly, the operator may have incorrectly identified some anatomical structures resulting in disorientation which may or may not be realized, and thirdly, the operator may just be "lost" with identification of some or all anatomy correctly resulting in realized disorientation. We believe the results of this study have relevance to all the variations of disorientation described above.

By exposing visual behaviour and by inference attention processes of surgeons, this study represents an initial step in attempting to decrease the effects of disorientation in minimally invasive surgery. This study raises some important questions. Firstly, can these common reorientation strategies be taught to aspiring surgeons as part of a curriculum thereby decreasing the learning curve associated with the apparent need for experience in laparoscopy? Secondly, can these common reorientation strategies be taught to individual surgeons who performed below average to increase their performance? Should this be the case, teaching reorientation strategies should be incorporated into the laparoscopic teaching curriculum for surgical trainees. The next step in the investigation of this paradigm will be to evaluate whether performance can be improved in a prospective, randomised controlled trial of teaching orientation strategies unveiled in this study. 


\section{References}

1. Khan MH, Howard TJ, Fogel EL, et al. Frequency of biliary complications after laparoscopic cholecystectomy detected by ERCP: experience at a large tertiary referral center. Gastrointest Endosc 2007; 65(2):247-52.

2. Karvonen J, Gullichsen R, Laine S, et al. Bile duct injuries during laparoscopic cholecystectomy: primary and long-term results from a single institution. Surg Endosc 2007; 21(7):1069-73.

3. Bektas H, Schrem H, Winny M, Klempnauer J. Surgical treatment and outcome of iatrogenic bile duct lesions after cholecystectomy and the impact of different clinical classification systems. Br J Surg 2007; 94(9):1119-27.

4. Veen EJ, Bik M, Janssen-Heijnen ML, et al. Outcome measurement in laparoscopic cholecystectomy by using a prospective complication registry: results of an audit. Int J Qual Health Care 2008; 20(2):144-51.

5. Hugh TB. New strategies to prevent laparoscopic bile duct injury--surgeons can learn from pilots. Surgery 2002; 132(5):826-35.

6. Dekker SW, Hugh TB. Laparoscopic bile duct injury: understanding the psychology and heuristics of the error. ANZ J Surg 2008; 78(12):1109-14.

7. Way LW, Stewart L, Gantert W, et al. Causes and prevention of laparoscopic bile duct injuries: analysis of 252 cases from a human factors and cognitive psychology perspective. Ann Surg 2003; 237(4):460-9.

8. ElHelw M, Nicolaou M, Chung A, et al. A gaze-based study for investigating the perception of visual realism in simulated scenes. ACM Trans. Appl. Percept. 2008; 5(1):1-20.

9. Yang GZ, Dempere-Marco L, Hu XP, Rowe A. Visual search: psychophysical models and practical applications. Image and Vision Computing 2002; 20(4):273-287.

10. Kahneman D. Attention and effort. Englewood Cliffs ; [Hemel Hempstead]: Prentice-Hall, 1973.

11. Chung AJ, Deligianni F, Hu XP, Yang GZ. Extraction of visual features with eye tracking for saliency driven $2 \mathrm{D} / 3 \mathrm{D}$ registration. Image and Vision Computing 2005; 23(11):999-1008.

12. $\mathrm{Hu}$ XP, Dempere-Marco L, Yang GZ. Hot spot detection based on feature space representation of visual search. IEEE Trans Med Imaging 2003; 22(9):1152-62.

13. Dempere-Marco L, Hu XP, MacDonald SLS, et al. The use of visual search for knowledge gathering in image decision support. Ieee Transactions on Medical Imaging 2002; 21(7):741-754.

14. Duchowski AT. Eye tracking methodology : theory and practice. 2nd ed. ed. London: Springer, 2007.

15. Sodergren MH, Orihuela-Espina F, Clark J, et al. A hidden markov modelbased analysis framework using eye-tracking data to characterise reorientation strategies in minimally invasive surgery. Cogn Process 2009.

16. Sodergren MH, Orihuela-Espina F, Clark J, et al. A model to characterise reorientation strategies in natural orifice translumenal endoscopic surgery. Cogn Process 2009; 10 Suppl 2:S307-11.

17. Jain AK, Murty MN, Flynn PJ. Data clustering: A review. Acm Computing Surveys 1999; 31(3):264-323. 
18. Hermer L, Spelke ES. A geometric process for spatial reorientation in young children. Nature 1994; 370(6484):57-9.

19. Hermer L, Spelke E. Modularity and development: The case of spatial reorientation. Cognition 1996; 61(3):195-232.

20. Hermer-Vazquez L, Spelke ES, Katsnelson AS. Sources of flexibility in human cognition: dual-task studies of space and language. Cogn Psychol 1999; 39(1):3-36.

21. Chiandetti C, Vallortigara G. Spatial reorientation in large and small enclosures: comparative and developmental perspectives. Cogn Process 2008; 9(4):229-38.

22. Posner MI, Snyder CR, Davidson BJ. Attention and the detection of signals. J Exp Psychol 1980; 109(2):160-74. 